\title{
Converting Motion between Different Types of Humanoid Robots Using Genetic Algorithms
}

\author{
Mari Nishiyama and Hitoshi Iba
}

\begin{abstract}
The imitation between different types of robots remains an unsolved task for a long time. The assignment of the correct angles to each joint is critical for robot motion. However, different robots have different structures, thus this discrepancy causes a difficulty when converting a motion to another type of robot. For solving this problem, we propose a GA-based method that can find the conversion matrix needed to map joint angles of one robot to another. There are two objectives to consider when creating an imitation; reducing the difference between the ideal imitation and the converted imitation and keeping the stability. Three experiments were conducted; a stable experiment, an unstable experiment and a double learning experiment. As a result, the double experiment showed a high concordance rate of $93.5 \%$, the highest stability and the fastest speed of all experiments. These results show great promise for the proposed method as a way to realize motion imitation between different types of robots.
\end{abstract}

Index Terms-Robot, imitation, motion planning, humanoid robot, genetic algorithms, autonomous learning.

\section{INTRODUCTION}

The control of robot motion has been researched from various aspects [1]-[3], taking a principal position in robotics. In these researches, humanoid robots are different from other robots such as wheeled mobile robots, since their structures are exceedingly complicated.

The fact that an enormous amount of each joint's angle data are needed to move humanoid robots makes it difficult to create motions of humanoid robots.

So far, studies on the imitation of robots' movements have mostly aimed for robots to imitate human motion [4]-[6].

However, the majority of these studies first simplifies the human's motion and then assigns this motion data to robots. These studies are limited in the sense that the degrees of freedom (DOF) or movable ranges of the robots' joints are not the same as the humans'. This makes it difficult to transfer the human's motion directly to the robot.

Tetsunari Inamura et al. [7] proposed a method for motion imitation between robots. However, this study was limited to the reproduction of motion between the same types of robots. Thus, conversion and imitation between robots that have different kind of joint structure have not been attempted yet, as far as we know.

This paper focuses on the imitation of motions between

Manuscript received March 11, 2014; revised May 10, 2014.

Mari Nishiyama is with the Department of Electrical and Electronic Engineering, the University of Tokyo, Japan (e-mail: nishiyama@iba.t.utokyo.ac.jp).

Hitoshi Iba is with the Graduate School of Information Science and Technology, the University of Tokyo, Japan (e-mail: iba@iba.t.utokyo.ac.jp). different kinds of robots.

Similar to the differences between joints of robots and joints of human, there is a difference in positions and movable ranges between joints of different types of robots. Moreover, there are some cases where the joint of one robot cannot be directly mapped to the joint of another kind of robot, since it lacks this specific joint. It is the aim of this research to find an optimal conversion matrix for motion imitation for converting joint angles from one robot to another, even if the structures of these two robots are completely different.

We estimated that the usage of GA would lead to a reduction of the amount of learning data required for the joint angles of the robot compared to other methods such as machine learning techniques, which needs a lot of training data. Consequently, using GA can reduce the manual involvement when creating the robot motion.

\section{PROPOSED METHOD}

This paper aims to verify whether it is possible to map joints between two different types of robots. To realize this objective, we searched for a conversion matrix able to map the model robot's actions to the imitating robot in such a way that they resemble each other as closely as possible. In this paper, the model robot is called a teacher robot, and the imitating robot is called a student robot.

Each joint angle of the student robot is assumed to be composed of a weighted combination of the joint angles in the teacher robot. The conversion matrix specifies the contribution from each of the teacher robot's joints. This allows neighboring joints to affect the resulting translation. It is natural that the joints in the arm are correlated. Thus, it is assumed to be important to consider the influence of the neighboring joints in order to convert a joint angle. We used a GA to find the best conversion matrix. The elements of the conversion matrix are illustrated in Fig. 1.

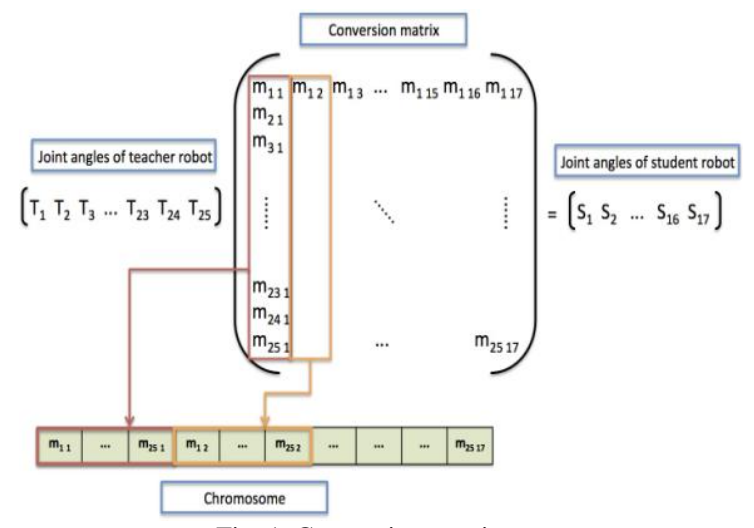

Fig. 1. Conversion matrix. 
When searching for the optimal conversion matrix for motion imitation, two objectives were considered; the similarity and the stability of the motion. A fitness function was developed for each of these objectives. Three methods of combining these fitness functions were then investigated.

The similarity evolution mainly considers the similarity of the imitation. Joint angle data for different motions were prepared, one or two training motions and one test motion. For the teacher robot, every motion was created manually, whereas only the training motions were needed for the student robot. When training the student robot, the conversion matrix was applied to the teacher robot's joint angle data at a specific moment in time. The converted joint angles of the student robot were then compared with the ideal imitation data, which had been created manually. The fitness function was defined such that the smaller the difference of the joint angles between the converted imitation and the ideal imitation the higher the fitness value. The conversion matrix that had the highest fitness value in the last generation was considered as the optimal solution. Then this optimal conversion matrix was applied to the test motion, that is a different from the training motion. The conversion matrix converted the test motion of the teacher robot, after which it was decided whether it had been able to properly imitate the teacher robot's motion.

The stability evolution considers the stability of the student robot that is whether or not the robot keeps standing during the motion. Kim et al. [8] investigated how to keep the stability when converting a motion from a human to a robot. This study used a complex calculation that confirmed the stability of the robot, both kinetically and dynamically. We aimed to simplify the process and evolve the stability of the student robot using GA.

As mentioned earlier, three different methods were used to evolve the conversion matrix. Each method is illustrated in Fig. 2. The first method, the similarity method, uses only a similarity measure as its fitness function. The second method, the mixed method, instead uses a fitness function that consists of a combination of the same similarity measure and a stability measure. These two measures are multiplied and thus both are considered during the whole evolution. The last method, the separated method, first finds the conversion matrix with the similarity method, but then continues to evolve the best individuals using the stability measure only.

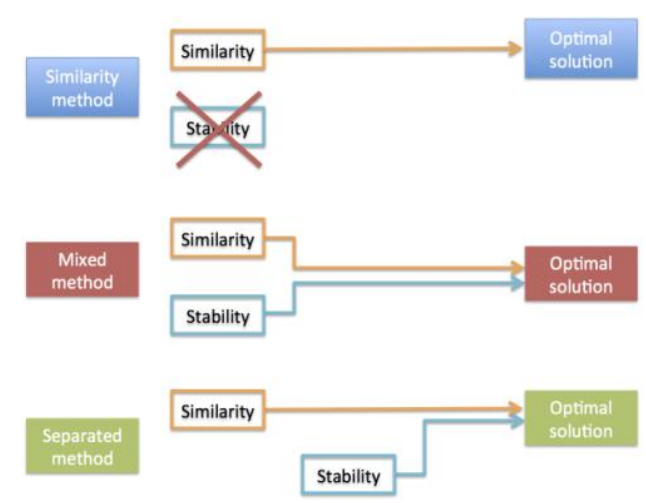

Fig. 2. Three methods

We assumed that the separated method would be the most effective. The reason is that the similarity of the motion is considered to be the most important factor at the beginning of the evolution. If the student robot can imitate the teacher robot's motion, the stability of the student robot will improve naturally. Considering the stability too early in the process might cause an alienation from the motion of the teacher robot, and the main objective of this study, i.e. to imitate motions, cannot be accomplished even if the student robot can stand.

\section{EXPERIMENT}

\section{A Experiment Environment}

HOAP-2[9], made by Fujitsu Automation Inc., was used as the teacher robot. KHR-2HV [10], made by Kondo Science Inc., was used as the student robot. HOAP-2 has 25 DOF and KHR-2HV has 17 DOF. HOAP-2 has an attitude sensor, two foot sensors and two USB cameras. KHR-2HV has an accelerate sensor and a gyro sensor. In this paper, we used both a robot simulator and a real robot. Webots PRO 7.0.1 [10] was used as the robot simulator.

These data were composed of time series data from zero seconds to five seconds, which were separated into eighty sections. The reciprocal of the difference between the student robot's joint angle data of the sample imitation and the student robot's joint angle data of the converted imitation was used as the fitness value. The fitness function thus performs a least-square error minimization.

The expression of fitness function in this experiment is as follows.

$$
f_{i}=\frac{1}{\sum_{k=1}^{N} \sum_{l=1}^{M}\left(t_{k l}-s_{k l}\right)^{2}}
$$

In this equation, $f_{i}$ is the fitness value of individual $i . N$ is the number of joints. $M$ is the time, $t_{k l}$ is the k:th joint angle of the student robot in the sample imitation at the time $l . s_{k l}$ is the $\mathrm{k}$ :th joint angle of the student robot in the converted imitation at the time $l$. The fitness function is defined such that the smaller the denominator, that is the difference between two joint angles, the higher the fitness value.

As a measure of the stability, we used a fitness value, which was in proportion to the time from the start of the simulation to the moment that the robot fell down, that is a chromosome that could stand for a long period of time was more likely to survive the evolution. The robot simulator can detect the position of each joint with its position sensor. Using this function, we could determine the time when the student robot should be considered to have fallen down. The fitness value was increased during the time when the value of sensors that showed the position of the neck joint remained above a certain threshold. If the position of neck joint fell under the threshold, the student robot was assumed to have fallen down and the fitness value would stop increasing. We used Webots to verify the stability of the robot. We made the student robot move with the converted motions in Webots.

In this paper, we conducted three experiments. The first experiment was a stable experiment. The objective of this experiment was to compare the three methods, the similarity method, the mixed method and the separated method. In the stable experiment, we attempted to make the student robot 
imitate a simple motion, in which only the arms were used. Four joints were used in the training motions for each robot in the stable experiment. The training motion was to raise and rotate their arms.

The second experiment was an unstable experiment. The objective of this experiment was to confirm the stability of the imitating motion created by the proposed method. Thus, we attempted to make the student robot imitate a kicking motion, which is an unstable motion. Since the foot area of the teacher robot is large enough to support its body stably only using one foot, the teacher robot can kick without moving its arms. On the other hand, the foot area of the student robot is smaller and the position of the center of gravity is higher than that of the teacher robot. Thus the student robot easily falls if it merely imitates the motion of the teacher robot. In the unstable experiment we aimed to verify that a stable imitation is achieved by the proposed method even if the original motion is unstable. Seven joints of the teacher robot and eight joints of the student robot were used as the training motions in the unstable experiment. In the training data, the robots was bending and stretching as they rotated their knees.

The third experiment was a double learning experiment. This experiment was conducted to investigate the effect of using multiple sets of training data. In this experiment, we used two sets of training data for learning. The training data used four arm joints. The test data of the teacher robot was the same as the one we used in the stable experiment.

\section{B Result}

\section{1) Stable experiment}

The results of each method in the stable experiment are shown in Fig. 3. This graph shows the change of the concordance rate that is the similarity between the joint angle data obtained by using the conversion matrix and the ideal imitation of the training data.

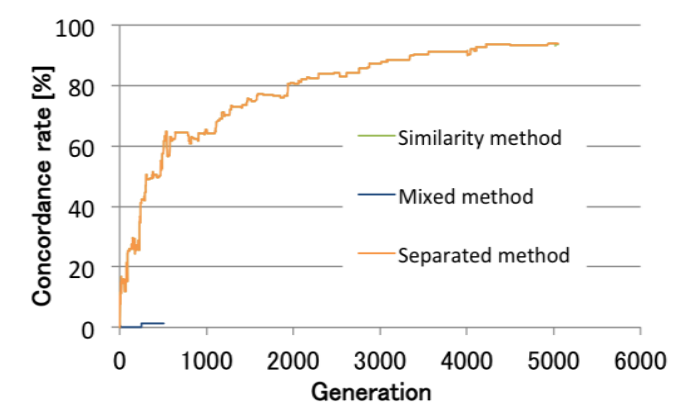

Fig. 3. Change of concordance rate in stable experiment.

The mixed method only achieved a very low concordance rate as seen in Fig. 3. It took around a week to calculate even 500 generations. The reason is that the stability evolution needs to use Webots, but moving robots in Webots is much slower than calculating the similarity only. Thus the calculation time of the mixed method was about ten times as long as that of the other two methods.

On the other hand, both the similarity method and the separated method achieved a higher concordance rate as the number of iterations increased. The difference between the similarity method and the separated method was the use of the stability evolution. In Fig. 3, the separated method still kept evolving for 50 generations even after the evolution using the similarity method has stopped. The similarity criterion is still used during the stability evolution. The similarity decreases during the stability evolution. However, the decline was only by a small amount. The final concordance rate was $93.7 \%$. The similarity method excelled the separated method in terms of the similarity of joint angles between the ideal imitation and the converted imitation. This difference is however not noticeable in the resulting motion. Keeping the balance of the student robot is more important than pursuing a small similarity improvement. Thus we concluded that the separated method was overall the best method.

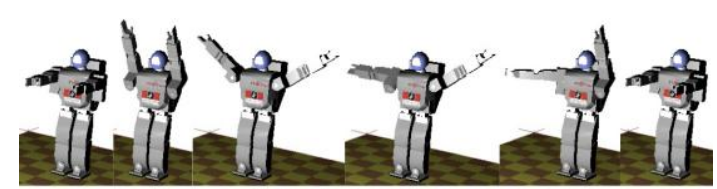

(A) Original motion

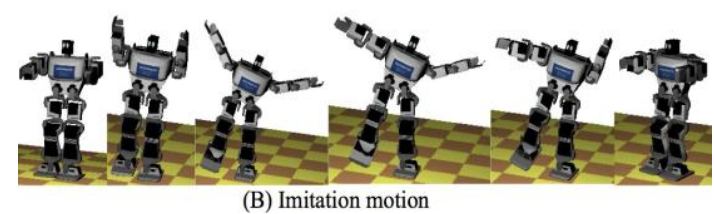

Fig. 4. Comparing original motion and imitation motion of stable experiment.

The teacher robot's motion and the imitation of the student robot converted from the conversion matrix evolved by the separated method are shown in Fig. 4. The arm motions derived from the optimal solution were similar to the original motion that was to be imitated. However, the leg motions were different from both the teacher robot and those obtained using the conversion matrix evolved by the similarity method.

We conducted experiments with the student robot and made it perform the motions not only in the simulator but also in the real world. We fed the imitation joint angles data that are shown in Fig. 4 into the real robot. The Fig. 5 shows that the real robot was less steady than the simulated one; however, the real robot still managed to avoid falling and kept standing during the motion.

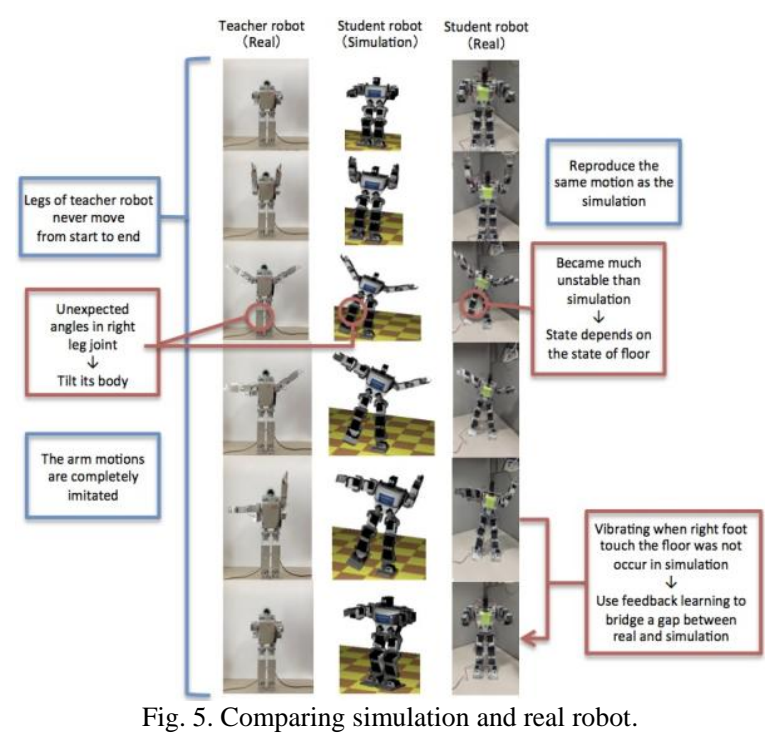

2) Unstable experiment

In the unstable experiment, we used the kicking motion as 
the original motion of the teacher robot shown in Fig. 5. This motion used not only arms but also legs, which is critical parts of body for the stability, thus it is much harder to remain stable during a kicking motion than a motion such as the one used in the stable experiment.

Using the similarity method, the resulted imitation fell down just after the simulation has started in the unstable experiment. To improve the stability, we tried the separated method and retrieved the result shown in Fig. 6. The student robot was able to keep upright longer than when the similarity method was used, although the stability was slightly worse than when the separated method was used in the stable experiment. As seen in Fig. 6, the resulting motion from the unstable experiment brings the student robot to lift its arm, which means that the fitness value in terms of the similarity has decreased. However, unlike the teacher robot, the student robot cannot lift one of its legs while keeping both arms straight because of its different structure. The student robot needs to keep its balance with its left arm raised in order to lift its right leg. Indeed, a sample motion that is attached to the student robot, KHR-2HV, also raises its left arm when it lifts its right leg. This fact shows the significance of the result of the unstable experiment.

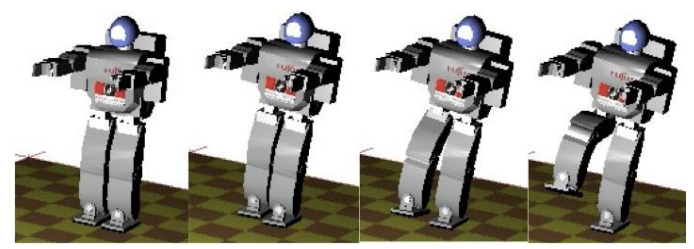

(A) Original motion

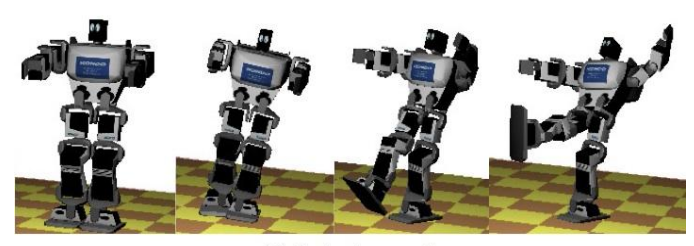

(B) Imitation motion

Fig. 6. Comparing original motion and imitation motion of unstable experiment.

The transition of the concordance rate that used training data in the unstable experiment is shown in Fig. 7.

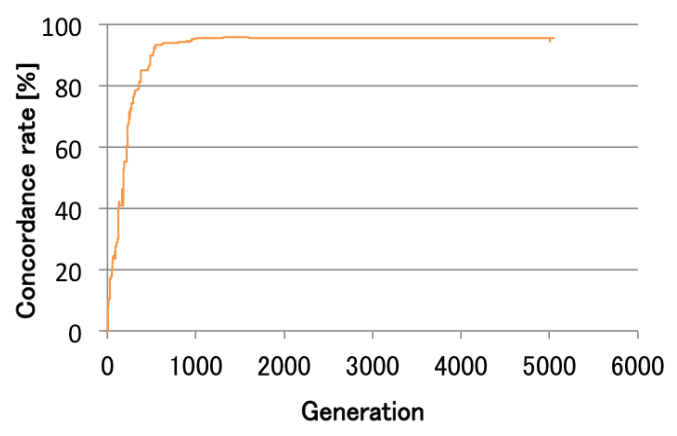

Fig. 7. Change of concordance rate in unstable experiment.

Although the rate decreased during the stability evolution, the decrease was limited to a small percentage. As a result, the final concordance rate was $95.5 \%$, which is slightly higher than that in the stable experiment.

\section{3) Double learning experiment}

In the double learning experiment, we used the same test motion as in the stable experiment. The aim was to confirm that evolution using multiple sets of training data is superior to learning using a single set of training data. The original test motion of the teacher robot, the resulting motion derived from the stable experiment (which used the separated method) and the resulting motion converted from the conversion matrix in the double learning experiment (which used only the similarity evolution) are compared in Fig. 8.

As seen in Fig. 8, the final motion imitation converted from the conversion matrix in the double learning experiment was the most accurate imitation of the teacher robot's motion. Moreover, the student robot in the double experiment succeeded to keep its stability without the stability evolution.

The concordance rate of the training data had changed as shown in Fig. 9.

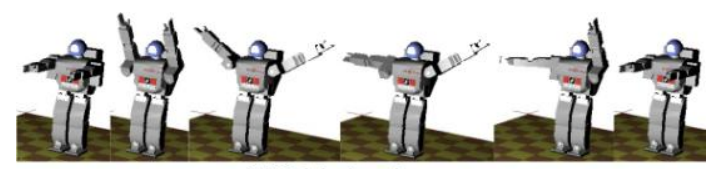

(A) Original motion

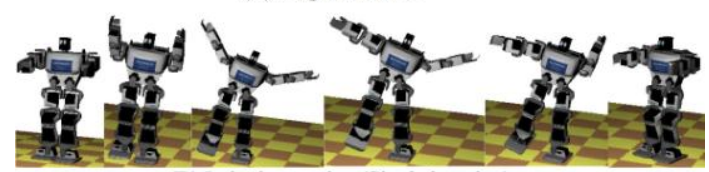

(B) Imitation motion (Single learning)

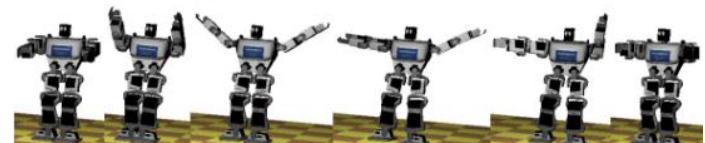

(B) Imitation motion (Double learning)

Fig. 8. Comparing original motion, imitation of stable experiment and double learning experiment.

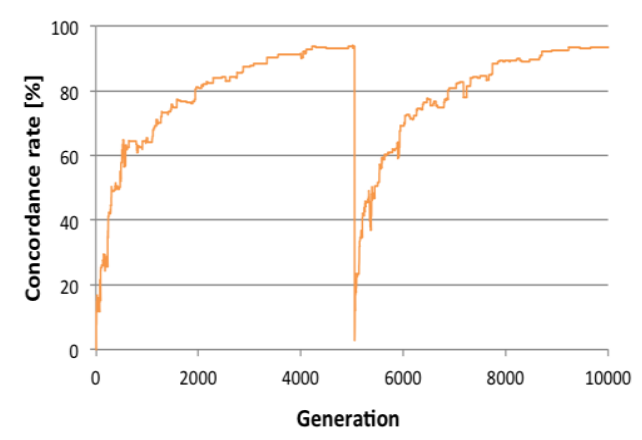

Fig. 9. Change of concordance rate in double learning experiment.

The place where the concordance rate decreased drastically is the point where we changed the training data from one to another. However, the concordance rate had risen gradually as the iterations had increased. The concordance rate of the final conversion matrix in the double learning experiment was $93.5 \%$. Moreover, since this optimal solution could keep the stability in the similarity evolution phase, the necessary time was only 0.136 seconds, which is about 19000 times faster than the separated method in the stable experiment.

\section{DISCUSSION}

In this paper, the student robot succeeded to imitate the teacher robot's motion by evolving the conversion matrix with GA. However, some joints moved unexpectedly in the 
converted motion imitation. This problem can have two different types of causes; one is the necessary motion introduced to keep the stability, which is seen in the unstable experiment. While these motions decreased the similarity, they played an important role in the motion imitation between two types of robots that have different structures. Converting motions while considering the difference between joint structures, and also how to compensate for unstable motions, is too complicated to realize manually. Here we can see the great benefits of using GA. Thus this type of unexpected motion can be seen as a positive factor.

The other type of unexpected motions causes instability. These should be avoided, but even so they were still some unstable motions present. There can be two explanations for this.

First, the fitness function may have been too simple to deal with this problem. In this paper, the expression (1) was used to calculate the fitness value in terms of the similarity. However, this expression minimizes the squared error, which is the most basic error estimation. Thus, it is hard to predicate whether or not this method is accurate. There probably exists another fitness function, which can calculate the fitness value more accurately. Moreover, we used the time length that the robot kept standing as a fitness value in terms of the stability. However, this is also very simple and might not enough to check its stability. We have to find another fitness function, such as considering the center of gravity of the robot.

Second, the learning was insufficient. In this paper, we compared the stable experiment, which used only one training motion and the double learning experiment, which used two training motions. As a result, we confirmed that using two sets of training data is superior to using one training data. Presumably, an increased amount of training motions would introduce even more diversity in the learning that would lead to a better estimate of the conversion matrix.

Based on these findings, there are four problems that are interesting for future work.

First, it is important to find the proper parameters for GA, such as the probability of mutation. In order to inspect what values that achieve the best results, the conversion matrix should also be evolved using as many different training motions as possible.

Second, we should consider the way to deal with the problem that some joints move unexpectedly in the negative sense. It does not seem strange for some joint angles to differ slightly from the training data. However, if the joint angles that did not move in the original motion starts to move in the motion converted by the conversion matrix the difference will be noticeable between the teacher robot's motion and the student robot's imitation. A difference in the joint angles of the legs also imposes a risk of falling. Thus, it is necessary to find a way to avoid giving mobility to joint angles, which did not move originally.

A concrete method to realize this purpose is to impose penalties in the fitness function. This will give individuals that introduce a motion to originally stationary joints a lower fitness value and thus reduce their change of survival. However, moving joints, which did not move in the manually constructed motion, is not always a bad result. As shown in the unstable experiment, it is sometimes useful to move unexpected joints in order to keep the balance. This kind of conversion process is hard to obtain if humans were to convert the joint angles manually. We have to consider a balance of both the similarity and the stability of the motion.

Third, we need to increase the number of the joints that are used for motions. In this paper, the maximum number of joints we used was only eight. However, the real robots have around twenty degrees of freedom. Most of the robots used in the real world also have over eight degrees of freedom for its movement. To be use of practical use, this method needs to deal with a larger data of joint angles.

Finally, we have to be able to reproduce the motion imitation stably in the real robot. As shown in the stable experiment, the motion, which moved stably in the simulation, is not always stable in the real world. While the environment in the simulation is ideal, the real world has a variety of environments such as a slopes, roughness and friction of floors. Considering the effects of these disturbances, it is desirable to find a robust conversion matrix that is not to be affected by disturbances by using machine learning such as the feedback training, for example, such as the reinforcement learning.

\section{CONClusion}

In this paper, we proposed a new method of motion imitation between two different types of robots. We searched for an optimal conversion matrix to convert the teacher robot's joint angles into the student robot's joint angles using GA. Two different approaches were considered in order to find a conversion matrix able to translate a motion from one type of robot to another, while keeping the balance; the similarity evolution and the stability evolution. We used three different methods to evolve the conversion matrix, the similarity method, the mixed method and the separated method. In order to investigate which method that was most suitable for the objective to imitate an initially stable motion, without losing the stability, we conducted the stable experiment. As a result, the separated method was confirmed to be best in these three methods as expected. The obtained matrix was then used to map an unseen motion from the teacher robot to the student robot, which was able to imitate the motion satisfactory, both in the simulation environment and in a real world setting.

In the unstable experiment, the robot also successfully found a way to keep its stability by using joints that were not used in the original motion of the teacher robot. It is hard for humans to find a conversion matrix taking the stability of the motion into account. According to the result of the unstable experiment, using not only the similarity evolution but also the stability evolution was very effective in this case.

However, we also managed to find a conversion matrix that created stable motion imitations without the stability evolution, in the double learning experiment. Since the stability evolution is much more time consuming than the similarity evolution, it is desirable to find a method that only uses the similarity evolution. Consequently, our proposed method that uses multiple training data during evolution shows good results in terms of the similarity, stability and efficiency and is thus considered optimal. 


\section{REFERENCES}

[1] S. Calinon, F. Guenter, and A. Billard, "On learning, representing and generalizing a task in a humanoid robot," IEEE Transactions on Systems, vol. 36, no. 2, 2007.

[2] R. A. Brooks, "A robust layered control system for a mobile robot," IEEE Journal of Robotics and Automation, vol. RA-2, no. 1, 1986.

[3] K. Hirai, M. Hirose, Y. Haikawa, T. Takenaka, and Honda R\&D Co., Ltd., "The development of honda humanoid robot," in Proc. IEEE International Conference on Robotics \& Automation, 1998, pp. 13211326.

[4] C. G. Atkeson and S. Schaal, "Robot learning from demonstration," in Proc. the Fourteenth International Conference, 1997, pp. 12-20.

[5] M. Mataric, "Getting humanoids to move and imitate," IEEE Intelligent Systems, pp. 18-24, 2000.

[6] S. Nakaoka, A. Nakazawa, K. Yokoi, H. Hirukawa, and K. Ikeuchi, "Generating whole body motions for a biped humanoid robot from captured human dances," in Proc. International Conference on Intelligent Robots and Systems, 2005, pp. 3157-3162.

[7] T. Inamura, Y. Nakamura, and I. Toshima, "Embodied symbol emergence based on mimesis theory," International Journal of Robotics Research, vol. 23, no. 4, pp. 363-377, 2004.

[8] S. Kim, C. Kim, B. You, and S. Oh, "Stable whole-body motion generation for humanoid robots to imitate human motions," in Proc. IEEE International Conference on Intelligent Robots and Systems, 2009, pp. 2518-2524.

[9] Miniature humanoid robot Hoap-2. (2013). [Online]. Available: http://biorob2.epfl.ch/pages/studproj/birg54248/hoap2catalog\_1.pdf
[10] KHR-2HV assembly instruction manual. (2013). [Online]. Available: http://www.kondokagaku.jp/data_download/KHR$2 \mathrm{HV} \backslash$ HardwareManual $\_$forENG.pdf

[11] Webots: Robot simulator. (2013). [Online]. Available: http://www.cyberbotics.com/

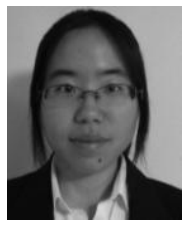

Mari Nishiyama was born in Tsukuba, Japan on December 30, 1990. She received the bachelor degree in engineering from the University of Tokyo in 2014. She studies robotics, genetic algorithms. She is a member of the Institute of Elect

rical Engineers of Japan.

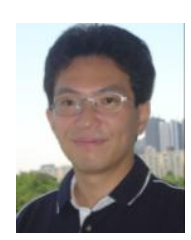

Hitoshi Iba received the Ph.D. degree from the University of Tokyo, Tokyo, Japan. From 1990 to 1998 , he was with the Electro Technical Laboratory, Ibaraki, Japan. He has been with the University of Tokyo since 1998. His research interests include evolutionary computation, genetic programming, bio-informatics, machine learning, robotics and vision. Dr. Iba is an associate editor of IEEE Transactions on Evolutionary Computation and the "Journal of Genetic Programming and Evolvable Machines (GPEM)". 